\title{
Cosmic molecular laboratories
}

\section{Commentary}

Production and investigation of molecules in our laboratories on earth are regular, exciting and challenging tasks. Quite often big efforts are involved in these tasks. Therefore, before 1963, scientists were against the opinion for existence of molecules in interstellar space. After the discovery of radio-window in 1932, the first molecule $\mathrm{OH}$ was discovered in the interstellar space in 1963. Though the discovery was against the opinion of scientists, it created lot of interests in the cosmic molecules. As of today, at least 252 molecules have been identified in the interstellar space. Some regions in the interstellar space are found to have a number of molecules and they may be called as the cosmic molecular laboratories. Because of the existence of $\gamma$-rays, x-rays and ultraviolet (UV) radiations in in-terstellar environment, scientists were against the opinion of existence of molecules in the interstellar space. Since the radiations produced by molecules, in general, cannot be seen by our eyes, it is not possible to see their existence directly in the interstellar space. In the year 1932, Karl Jansky discovered that the radiations from the inter-stellar space, having long wavelengths, also reach the earth's surface. This discovery opened a new astronomical world, known as the radio-window, having radiations from few $\mathrm{mm}$ to decametre in wavelength. After the discovery of radio-window, scientists had opinion that if any molecule exists in the interstellar space, we should get its radiations through the radio-window.

For three decades, after the discovery of the radio-window, no evidence about the existence of molecules in the interstellar space was found. In the year 1963, the first molecule $\mathrm{OH}$ was identified through absorption of $18-\mathrm{cm}$ radiation. Though this finding was surprising, as the belief of scientists shattered down, but they got interested in the search of more molecules in the interstellar space, and as of today at least 252 molecules are identified there. From the interstellar space, we get only radiations. For identification of a molecule in the interstellar space, astronomers depend very much on the spectroscopic study of the molecule performed in a terrestrial laboratory. In a terrestrial laboratory, the relative velocity between the source and the observer is zero and for a given molecule $\mathrm{M}$ the measured wavelengths are known as the rest-wavelengths. In the scenario of expanding universe, the same molecule $M$ present in a cos- mic object (a region in the interstellar space) provides radiations of Doppler- shifted wavelengths. These radiations bring information about the physical conditions (ki-netic temperature, pressure, density etc.) prevailing in an astronomical object and about the chemical reactions going on there. Molecules are found associated with the star- forming regions and in the circumstellar envelopes of evolved stars. Thus, the study of molecules provides valuable information about the physical conditions prevailing at the time of the birth of a star and at the time of the death of a star.

The earth's atmosphere is transparent for two bands in electromagnetic spectrum, known as the (i) optical-window, and (ii) radio-window. The average wavelength of radiations in the radio-window is about $10^{5}$ larger than that in the opticalwindow. Consequently, the scattering of radiations in the radio-
Volume I Issue 2 - 2017

\author{
Mohit Kumar Sharma, Suresh Chandra \\ Amity Center for Astronomy \& Astrophysics, Amity University, \\ India
}

Correspondence: Suresh Chandra, Amity Center for Astronomy \& Astrophysics, Amity Institute of Applied Sciences, Amity University, India, Tel +91-9818005663, Email schandra2@amity.edu

Received:September 12, 2017 | Published: September 25, 2017

window is $10^{-20}$ times smaller than of those in the opticalwindow. Thus, in the context of distant cosmic objects (situated at a distance of, say, several hundred light- years), the radio-window plays very important role. The molecules produce the radiations in the radio-window due to transitions between the rotational levels. Molecular laboratories in the interstellar space are rather cool regions having kinetic temperature of few tens of Kelvin. There are about 47 regions (laboratories) in the interstellar space where the molecules are found. However, the largest number of molecules are found in 5 cosmic laboratories. The positions on the celestial sphere of these laboratories and their distances from the earth are given in Table 1. (The light-year, denoted by ly, is equal to the distance traveled by light in one year in vacuum. 1ly $=9.5 \times 1015 \mathrm{~m}$.).

While one has to put lot of efforts for production of molecules in the terrestrial laboratories, the nature is maintaining a number of molecular laboratories. having large number of molecules, the cosmic laboratories are not less important as com-pared to the terrestrial ones. Some molecules in the cosmic laboratories have shown MASER (microwave amplification by stimulated emission of radiation) action (nat- ural masers). LASERs and MASERs on the earth are quite artificial devices and a lot of efforts are required for their production. Another anomalous phenomenon shown by some cosmic molecules is the anomalous absorption (absorption against the cosmic microwave background). In this phenomenon the brightness temperature shown by radiation is less than the cosmic microwave background temperature $(2.73 \mathrm{~K})$. This anomalous absorption phenomenon could not be produced in the terrestrial laboratories. The authors are grateful to Hon'ble Dr. Ashok K.Chauhan, Founder President, and Hon'ble Dr. Atul Chauhan, Chancellor, Amity University for valuable support and encouragements.

\section{Acknowledgemtns}

None.

\section{Conflicts of interet}

The author declares there is no conflcit of interest. 
Table I The positions on the celestial sphere of laboratories and their distances from the earth

\begin{tabular}{llll}
\hline Object & Right ascension & Declination & Distance from Earth \\
IRC+102I6 & $9^{\mathrm{h}} 47^{\mathrm{m}} 57.4^{\mathrm{s}}$ & $13^{\circ} 16^{\prime} 43.56^{\prime \prime}$ & $390-490 \mathrm{ly}$ \\
TMC-I & $4^{\mathrm{h}} 4 \mathrm{I}^{\mathrm{m}}$ & $25^{\circ} 52^{\prime}$ & $450 \mathrm{ly}$ \\
Sgr B2 & $17^{\mathrm{h}} 47^{\mathrm{m}} 20.4^{\mathrm{s}}$ & $-28^{\circ} 23^{\prime} 7^{\prime \prime}$ & $390 \mathrm{ly}$ \\
Orion Nebula & $0^{\mathrm{h}} 22^{\mathrm{m}} 29^{\mathrm{s}}$ & $5^{\circ} 23^{\prime} 28^{\prime \prime}$ & $1344 \mathrm{ly}$ \\
W49 & $19^{\mathrm{h}} 10^{\mathrm{m}} 17^{\mathrm{s}}$ & $9^{\circ} 06^{\prime}$ & $45-48 \mathrm{ly}$ \\
\hline
\end{tabular}

